Interview

\title{
Interview with Professor Zhe-Sheng (Jason) Chen, Editorial Board Member of Med One
}

Med One Editorial Office

1st Floor Cobham MSA, Cobham, Surrey, Greater London, KT11 3DB, United

Kingdom

We are honored to have this interview with Professor Zhe-Sheng (Jason) Chen from St. John's University, who is currently serving as editorial board member of Med One.

\section{INTRODUCTION}

Professor Zhe-Sheng (Jason) Chen obtained a doctorate (Ph.D.) in Pharmacology from Kagoshima University, Japan. Dr. Chen also holds an MD degree from Guangdong Medical and Pharmaceutical College and a Master's degree in Toxicology from Sun Yat-Sen Medical University, China. Currently Dr. Chen is a Professor at St. John's University College of Pharmacy and Health Sciences. Dr. Chen is also the Director of Institute for Biotechnology at St. John's University. He has published more than 250 scientific publications. Dr. Chen is the founding Editor-in-Chief for Journal of Cancer Research Updates and is also the Editor-in-Chief for Journal of New Developments in Chemistry, as well as an editor of African Journal of Pharmacy and Pharmacology. He is an editorial board member of 26 journals and a reviewer of more than 220 peer-reviewed journals. As a consultant to pharmaceutical, biotechnology and health industry organizations, Dr. Chen commands invitations for more than 250 presentations, keynote addresses, and conference chairman. Dr. Chen is also a grant reviewer for the National Institutes of Health (NIH, USA), Chinese National Natural Science Foundation (China), the Genesis Oncology Trust (New Zealand), National Science Centre (Poland), the Government of the Hong Kong Special Administrative Region (HKSAR)

\section{Open Access}

Received: 15 May 2019

Published: 04 July 2019

Copyright (c) 2019 by the author(s). Licensee Hapres, London, United Kingdom. This is an open access article distributed under the terms and conditions of Creative Commons Attribution 4.0 International License. Grant (Hong Kong, China), the Hungarian Scientific Research Fund (OTKA) (Hungary), the Netherlands Organisation for Scientific Research (NWO) (Netherlands), and the Czech Science Foundation (Czech).

\section{INTERVIEW}

\section{Could You Describe to Us Your Current Research Interests?}

Our laboratory is interested in the molecular and cellular pharmacology of anticancer drugs, particularly drug resistance mechanisms and the reversal of such resistance, and new drug development. A philosophy of our research program is that progress in the chemotherapy of cancer will depend on rational combination of agents that reduce the function of 
plasma membrane transporters such as P-gp, ABCG2 and MRPs, and to enhance the actions of cytotoxic drugs. In collaboration with many researchers in USA, China, Japan, Canada, Australia and Egypt, studies in our laboratory focus on screening and identifying new inhibitors of $\mathrm{ABC}$ transporters via the in vitro membrane vesicles transport assay system and cell-based assay systems. Another research direction is to study the role of $A B C$ transporters family on the detoxification of chemicals (including carcinogens and mutagens) using knock-out mouse models. In addition, I enjoy being active in public service with both local and global impact.

\section{How Serious Do You Think the Drug Resistance Problem Is?}

Drug resistance represents a major obstacle to the treatment of cancer. Without its reversal, discovery of new therapeutic compounds will all inevitably fall to the wayside. Understanding the drug resistance mechanisms as well as knowing how to reverse the drug resistance may provide insights that may lead to improvements in the use of currently available anticancer drugs.

\section{What Is the Current Status of Anti-Cancer Drug Development?}

In recent years, more anticancer drug development is on targeted therapy, spanning from small molecule anticancer drugs to immunotherapy. It is in this exciting time that focus is critical to the success of anticancer drug development, and we need to be mindful of the nuances that exist in the pathophysiology of each cancer type, rather than aiming solely for a "silver bullet".

\section{What Is the Future Direction of Anti-Cancer Drugs?}

The future directions of anticancer drugs hold the potential to be more selective, with increased efficacy and less side effects. It is incredibly rewarding to be in a field where currently the focus is not only towards a cure but also in providing patients with improved quality of life.

\section{What Do You Want to Achieve with Your Research?}

Our primary goal is to further understand the drug resistance mechanisms and know how to reverse the resistance and eventually lead to improvements in the use of currently available anticancer drugs.

\section{What Has Been Your Most Important Scientific Finding? Your Most Surprising Finding?}

Our most important scientific findings are from our various studies of how $\mathrm{ABC}$ transporters play an important role in certain physiologic functions and in anticancer drug resistance. One of my most surprising findings to date is of Viagra being able to enhance anticancer drug sensitivity in some drug-resistant tumors! That may be a second 
"accidental" discovery of Viagra, first being the side effect that led to its most well-known indication.

\section{Could You Tell Us Who the Most Important Person in Your Career Is?}

I must sincerely thank the most important person that affected to my career, which is Professor Shin-Ichi Akiyama, my PhD mentor at Kagoshima University, Japan. Without a doubt, he was instrumental in initially developing me to be the scientist that I am today.

\section{Do You Have a Role Model in Science?}

My scientific role model is my late postdoc mentor, Dr. Gary D. Kruh (Fox Chase Cancer Center), as he contributed all his life to science.

You Had Many Editorial Experiences in the Past. What Do You Think Is the Most Interesting/Rewarding Part of Being an Academic Journal Editor?

In serving as an editorial member, the most interesting or rewarding part for me is the hard work and effort that come into fruition when publishing a good article. It is also eye-opening to me to see all the interesting research being presented globally.

\section{What Are the Characteristics of a Good Journal?}

A good journal should have high impact, easy access (free or with reasonable cost), operating a relatively smooth submission process without a high fee, and a solid peer-review community served by respectable scientists.

\section{Would You Recommend Your Colleagues to Serve on Journals' Editorial Board? Why?}

Yes, because serving on a journal's editorial board is a good experience for scientists. The time spent is well-rewarded by keeping us scientists informed with new discoveries. It is also a way for us to give back to the community that fostered us.

\section{Do You Have Any Suggestions for Early Career Researchers?}

For early career researchers, you should seek any opportunity to serve as reviewers or editorial board members. I received many emails to ask for reviewing articles from early career researchers, and later they asked me to send them letters of certificate as they need them for looking for a job, USA permanent resident (green card) applications, or for promotion. This is understandable, however, most of them stop to review articles because they told me that they already got their green cards, promotion was done, or already got a good job in a company. I think that this is not good behavior for responsible scientists, as good scientists do not review articles only for gaining their green cards in USA, promotions or good 
positions, but also really enjoy reviewing scientific articles. A successful career can only be built by actively engaging with and contributing to the scientific community.

How to cite this article:

Med One Editorial Office. Interview with Professor Zhe-Sheng (Jason) Chen, Editorial Board Member of Med One.

Med One. 2019;4:e190012. https://doi.org/10.20900/mo.20190012 